ARTIGO ORIGINAL ORIGINAL ARTICLE
Palavras-chave:

obinutuzumabe, leucemia linfoide crônica, custo-efetividade
Keywords:

obinutuzumab, chronic lymphocytic leukemia cost-effectiveness

\section{Análise de custo-efetividade de obinutuzumabe associado à quimioterapia para o tratamento de pacientes adultos com leucemia linfoide crônica não elegíveis ao tratamento baseado em fludarabina na perspectiva do Sistema Único de Saúde (SUS)}

\author{
Cost-effectiveness analysis of obinutuzumab associated \\ to chemotherapy for the treatment of chronic lymphocytic \\ leukemia patients ineligible for fludarabine under \\ the Brazilian Public Healthcare System (SUS) \\ Rodrigo Shimabukuro Ho', Camila Rufino Souza', Carolina Terumi Tsuchiya', \\ Juliana Marques Lagrasta Biondo', Marcia Regina Dias Alves' \\ DOI: 10.21115/JBES.v9.suppl1.41-48
}

\begin{abstract}
RESUMO
Objetivo: $O$ objetivo deste estudo foi avaliar a custo-efetividade da terapia obinutuzumabe + quimioterapia (GQT) versus quimioterapia (QT) em pacientes com leucemia linfoide crônica (LLC) sem tratamento prévio classificados como inelegíveis à dose completa de fludarabina (slow-go) na perspectiva do Sistema Único de Saúde (SUS). Métodos: Um modelo de Markov foi desenvolvido para acompanhar os pacientes com LLC durante o curso da doença, em um horizonte de tempo de 20 anos. Os desfechos de sobrevida livre de progressão (SLP) e sobrevida global (SG) foram avaliados respectivamente em termos de anos de vida livres de progressão (AVLP) e anos de vida ganhos (AVG). O custo de tratamento incluiu os custos de aquisição de medicamentos, manejo de eventos adversos e acompanhamento. Os dados de eficácia foram obtidos dos estudos CLL11 e CLL5. Resultados: O custo de tratamento incremental foi de R\$ 72.565. Os valores de SLP para GQT e QT foram, respectivamente, 3,3 e 1,1 AVLP. Para SG, o GQT resultou em uma efetividade de 5,7 e QT 4,3 AVG. Os resultados de RCEl foram de R\$32.477/SLP e R\$ 52.252/AVG. Conclusão: A terapia GQT é uma opção que proporciona benefícios clínicos superiores quando comparada à QT e pode ser considerada custo-efetiva no tratamento de LLC em pacientes não elegíveis a doses completas de fludarabina.
\end{abstract}

Recebido em: 17/07/2017. Aprovado para publicação em: 08/08/2017

1. F. Hoffmann-La Roche Ltd., São Paulo, SP, Brasil.

Instituição onde o trabalho foi executado: F. Hoffmann-La Roche Ltd. e SENSE Company.

Financiamento: $O$ estudo foi financiado pela F. Hoffmann-La Roche Ltd.

Autor correspondente: Marcia Regina Dias Alves. Av. Engenheiro Billings, 1729, Jaguaré, São Paulo, SP, Brasil. CEP: 05321-900

E-mail: marcia.alves@roche.com 


\section{Introdução}

A leucemia é uma doença caracterizada pelo acúmulo de células neoplásicas na medula óssea. A doença pode ser classificada como aguda quando se agrava rapidamente ou crônica quando seu desenvolvimento é lento. Adicionalmente, as leucemias podem ser classificadas de acordo com o tipo de leucócito afetado. Quando os linfócitos são acometidos, a leucemia é denominada linfoide, linfocítica ou linfoblástica. Caso as células mieloides sejam afetadas, a leucemia é denominada mieloide ou mieloblástica (Ministério da Saúde, Brasil. Instituto Nacional de Câncer José Alencar Gomes da Silva INCA, 2015).

A leucemia linfoide crônica (LLC) é definida pela proliferação clonal e acúmulo de linfócitos $B$ neoplásicos que geralmente expressam a molécula CD5+, no sangue periférico, medula óssea, baço e linfonodos (Cramer \& Hallek, 2011; Rozman \& Montserrat, 1995).

Em termos epidemiológicos, a LLC corresponde a 40\% de todas as leucemias em adultos acima de 65 anos de idade (Herishanu \& Polliack, 2005). Em países ocidentais, a LLC é a leucemia mais comum (Hallek, 2015) e a idade média para o diagnóstico é de 72 anos, com apenas 10\% dos pacientes sendo diagnosticados com menos de 55 anos de idade (Eichhorst et al., 2011).

A terapia combinada de fludarabina, ciclofosfamida e rituximabe (FCR) é considerada como padrão terapêutico em primeira linha para pacientes com LLC fisicamente ativos, sem outros problemas da saúde e com função renal normal (Eichhorst et al., 2011). Entretanto, a toxicidade excessiva, como infecções severas, não permite que a maioria dos pacientes idosos continue o tratamento (Shvidel et al., 2003). Portanto, a combinação de FCR não é recomendada para pacientes idosos que apresentam comorbidades e alteração de função renal (Hillmen et al., 2014). Para esses pacientes, classificados como slow-go, o tratamento recomendado são as terapias anti-CD20 associadas a doses moderadas de clorambucila (Hallek, 2015).

Obinutuzumabe é um anticorpo monoclonal humanizado recombinante tipo II anti-CD20 do isotipo IgG1, modificado pela glicoengenharia. O seu mecanismo de ação envolve a indução da morte celular direta e é um mediador de citotoxicidade celular dependente de anticorpos (CCDA) e fagocitose celular dependente de anticorpos (FCDA) (Produtos Roche Químicos e Farmacêuticos S.A., 2015). A sua eficácia e segurança foram demonstradas pelo estudo de fase III, CLL11, que comparou as terapias: obinutuzumabe e clorambucila (GClb); rituximabe e clorambucila (RClb) e monoterapia de clorambucila em 781 pacientes com LLC, previamente não tratados e não elegíveis a doses completas de fludarabina.

Os resultados do estudo demonstraram superioridade da terapia GClb com uma melhora significativa da sobrevida global (SG) e sobrevida livre de progressão (SLP) em relação a Clb (Tabela 1).
Tabela 1. Dados clínicos de GClb versus Clb (estudo CLL11)

$\mathrm{GClb}$ versus $\mathrm{Clb}$

\begin{tabular}{ll}
\hline Sobrevida global (SG) & $H R=0,62 ;$ IC95\%: 0,42-0,92; $p=0,0167$ \\
\hline $\begin{array}{l}\text { Sobrevida livre de } \\
\text { progressão (SLP) }\end{array}$ & $H R=0,20 ;$ IC95\%: 0,15-0,26; $p<0,0001$ \\
\hline
\end{tabular}

Atualmente, a única terapia disponível para o tratamento de LLC em pacientes não elegíveis à dose completa de fludarabina no SUS é apenas a quimioterapia (QT).

O objetivo do presente estudo foi avaliar a relação de custo-efetividade da terapia obinutuzumabe + quimioterapia (GQT) versus QT em pacientes com LLC, não tratados previamente e não elegíveis ao tratamento com dose completa de fludarabina, na perspectiva do Sistema Único de Saúde (SUS).

\section{Métodos}

Um modelo de sobrevida compartimentado foi elaborado com o intuito de acompanhar os pacientes com LLC ao longo do curso da doença até o final de suas vidas, em um horizonte temporal de até 20 anos (lifetime), assumindo-se ciclos semanais de transição entre os estados do modelo. Os estados de saúde foram definidos como sobrevida livre de progressão (SLP), progressão (refratário ou mudança de linha de tratamento) e morte, sendo que todos os pacientes iniciavam o modelo a partir do estado de SLP. Adicionalmente, no estado de saúde de SLP, o paciente podia transitar entre dois estados: em tratamento ou sem tratamento (Figura 1).

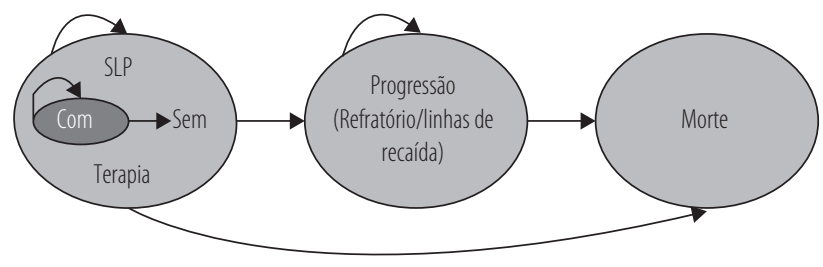

Figura 1. Esquema do modelo de Markov.

Os pacientes poderiam receber até seis ciclos de tratamento, a menos que a progressão fosse previamente confirmada ou que o tratamento tivesse que ser descontinuado devido à toxicidade, conforme o estudo CLL11.

\section{População}

A idade inicial dos pacientes que iniciaram o modelo foi de 72 anos, média dos pacientes do estudo CLL11. O peso e a altura adotados para um paciente foram de aproximadamente $67 \mathrm{~kg}$ e 1,65 m, conforme média populacional brasileira, de acordo com os dados do Instituto Brasileiro de Geografia e Estatística (IBGE) (Instituto Brasileiro de Geografia e Estatística - IBGE, n.d.). 


\section{Probabilidade de transição}

As probabilidades de transição no modelo foram extraídas do estudo CLL11 (Goede et al., 2015), a partir das curvas de SLP e de SG do estágio 1a correspondentes a comparação de GClb com Clb. As curvas de SLP e a de SG foram utilizadas até o tempo de observação estipulado no estudo clínico. Após esse período, as curvas foram projetadas por 20 anos (lifetime), por meio de uma distribuição de log-logística, opção paramétrica com melhor ajuste dos dados (Figura 2).

As taxas de progressão de SLP para morte foram calculadas com base nas taxas de mortalidade brasileira - de acordo com a idade da população geral (Instituto Brasileiro de Geografia e Estatítica - IBGE, 2012) - e as taxas de morte de SLP observadas para população do estudo CLL11. O modelo utilizou o maior valor entre as duas taxas, assumindo-se que os pacientes com LLC não apresentariam me- nor mortalidade do que a média populacional de mesma idade.

Já a probabilidade de mortalidade pós-progressão (transição do estado de progressão para morte) foi calculada com os dados de SG extraídos das curvas do estudo CLL5, com base na homogeneidade das populações de pacientes dos estudos CLL5 e CLL11. No estudo CLL5 (Eichhorst et al., 2009), a fludarabina foi comparada a clorambucila. Ao utilizar o tempo de sobrevida pós-progressão, a distribuição paramétrica foi ajustada para estimar a probabilidade de transição para morte, sendo a distribuição exponencial a que proporcionou melhor ajuste aos dados (Figura 3).

Além disso, outros dados clínicos, como a incidência de eventos adversos, foram adicionados ao modelo. As frequências de ocorrência foram obtidas do estudo CLL11, e apenas os EAs de grau 3 ou maior foram incluídos ao custo de tratamento (Goede et al., 2015) (Tabela 2).

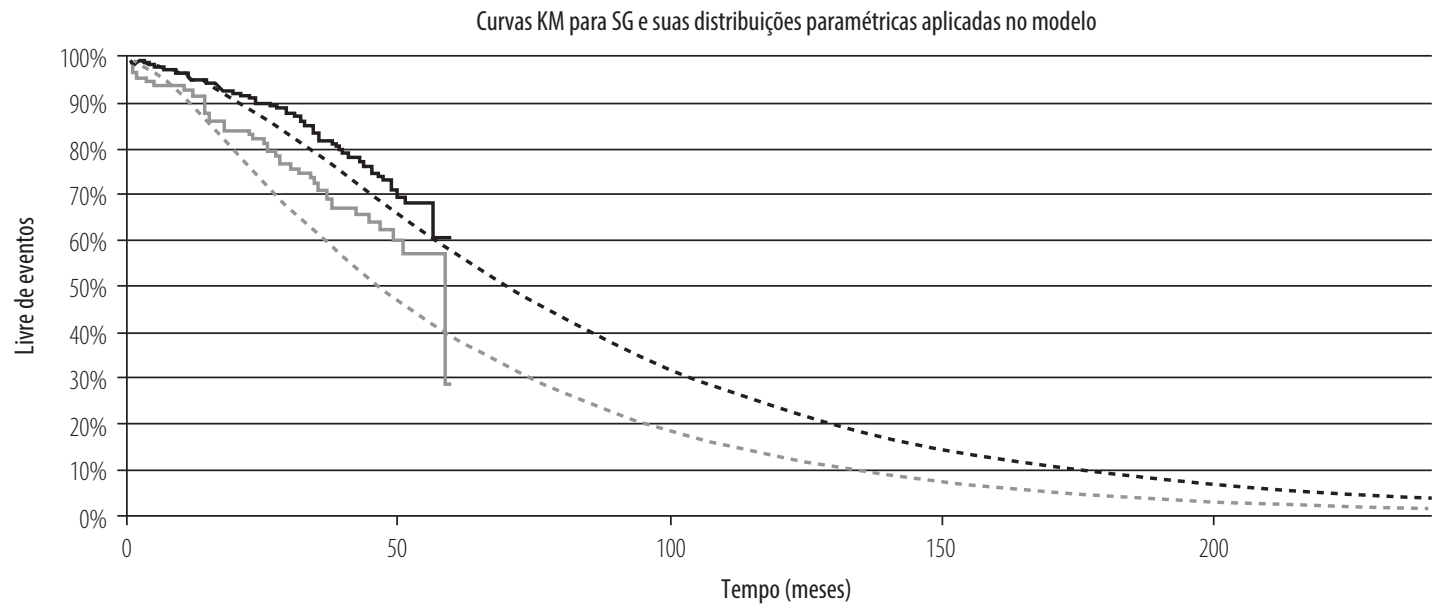

— KM SG GClb — KM SG Clb . . . . Modelo SG GClb . . . . Modelo SG Clb

Curvas KM para SLP e suas distribuições paramétricas aplicadas no modelo

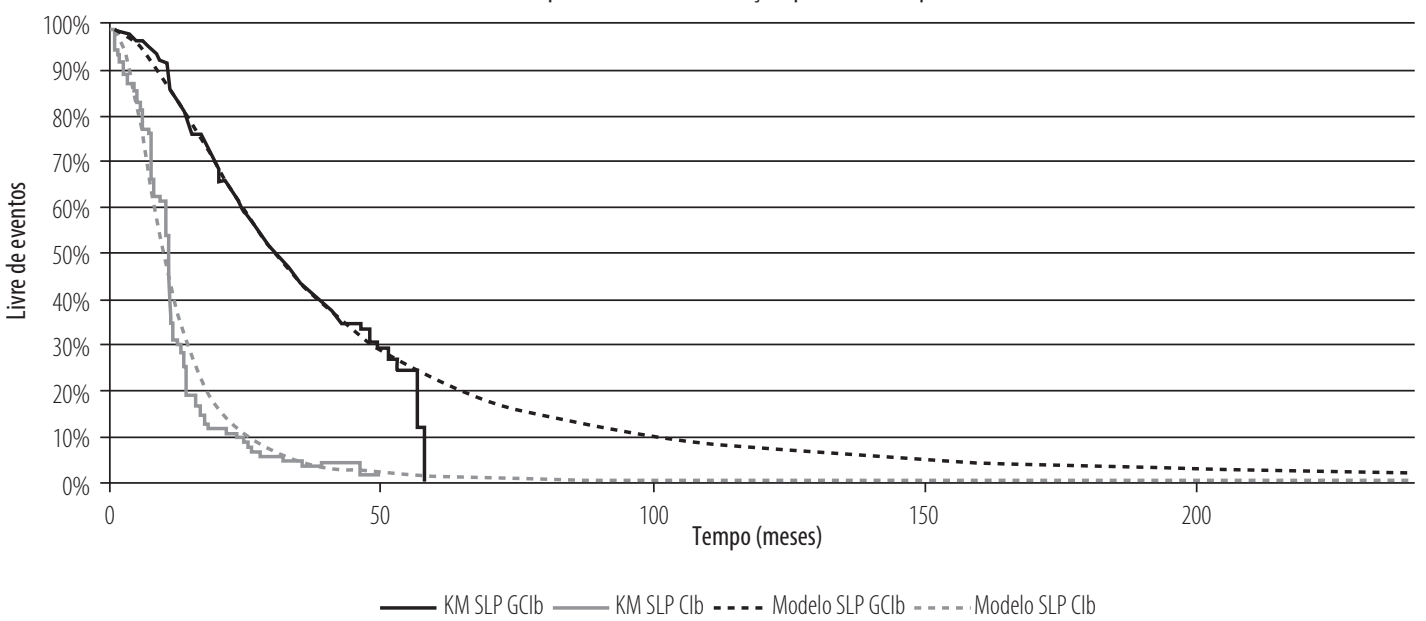

Figura 2. Curva de Kaplan-Meier - Extrapolação de SLP e SG utilizando modelo de distribuição loglogística (Estágio 1). 


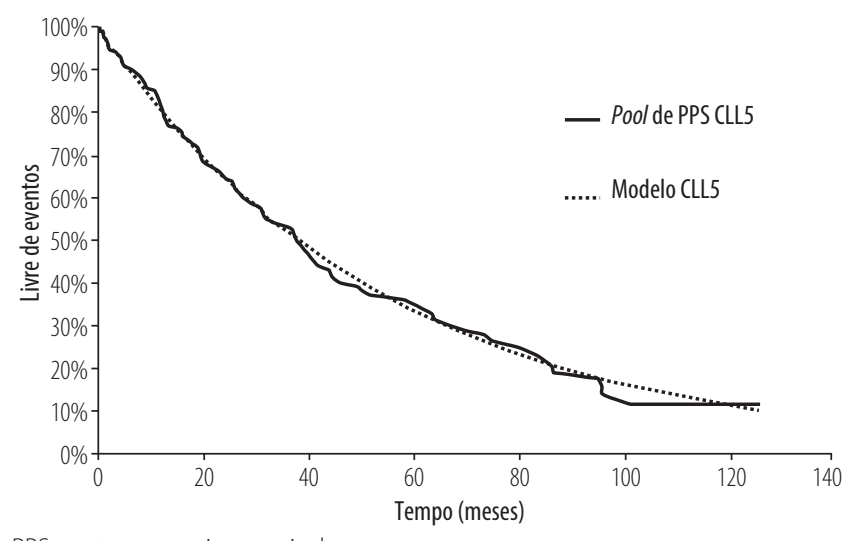

PPS: post-progression survival.

Figura 3. Extrapolação da curva pós-progressão do estudo CLL5.

Tabela 2. Incidência de eventos adversos

\begin{tabular}{lcc}
\hline Eventos & $\begin{array}{c}\text { GClb } \\
\text { (Estágio 1) }\end{array}$ & $\begin{array}{c}\text { Clb } \\
\text { (Estágio 1) }\end{array}$ \\
\hline Anemia & $5 \%$ & $4 \%$ \\
\hline Neutropenia febril & $2 \%$ & $4 \%$ \\
\hline Infecção & $11 \%$ & $14 \%$ \\
\hline Reação relacionada à infusão & $21 \%$ & - \\
\hline Leucopenia & $5 \%$ & $0 \%$ \\
\hline Neutropenia & $35 \%$ & $16 \%$ \\
\hline Pneumonia & $3 \%$ & $3 \%$ \\
\hline Trombocitopenia & $11 \%$ & $4 \%$ \\
\hline
\end{tabular}

Clb: clorambucila; GClb: obinutuzumabe + clorambucila.

\section{Uso de recursos e custos}

No estudo, apenas os custos médicos diretos (aquisição de medicamento, administração, acompanhamento e eventos adversos) foram contemplados na análise. Os custos indiretos e custos não médicos diretos não foram incluídos.

As posologias dos medicamentos foram baseadas nas bulas registradas e aprovadas, como exemplificados na Tabela 3 (Produtos Roche Químicos e Farmacêuticos S.A., 2015).

O custo unitário de obinutuzumabe foi obtido de acordo com a lista de preços da Câmara de Regulação do Mercado de Medicamentos (CMED) (Ministério da Saúde - Brasil.
Agência Nacional de Vigilância Sanitária - ANVISA, 2016), considerando o preço máximo de venda ao governo com 0\% de ICMS (PMVG 0\%). Para o custo de quimioterapia, a Autorização de Procedimento de Alta Complexidade (APAC) para o tratamento de LLC em primeira linha (APAC 03.04.03.005-8) foi considerada por representar o valor atualmente reembolsado pelo governo federal (Tabela 3) (Ministério da Saúde Brasil, 2016).

O custo de administração foi baseado na APAC 03.04.05.031-8 (monoquimioterapia do carcinoma de mama HER-2 positivo em estádio 3), que, apesar de tratar-se de outra indicação, considera apenas os custos de administração do medicamento, uma vez que o fármaco utilizado não tem seu valor contemplado na APAC (Ministério da Saúde - Brasil, 2011).

O custo de acompanhamento considerou apenas os custos de monitorização e acompanhamento do médico do paciente (Tabela 4).

\section{Custos de eventos adversos}

Os custos de eventos adversos foram obtidos por meio de microcusteio com padrão de uso de recursos definido pela opinião de especialistas, conforme demonstrado na Tabela 5.

\section{Outros custos}

Os custos no estado de SLP e progressão da doença foram adicionados ao modelo. Para o estado de SLP, apenas os custos de monitorização do paciente foram considerados no cálculo. Já para os custos na progressão da doença, a APAC de tratamento de segunda linha de LLC (03.04.03.006-6) foi considerada, correspondendo ao valor de $\mathrm{R} \$ 1.800,00$.

\section{Desconto}

Uma taxa de desconto anual de 5\% para custos e desfechos foi aplicada, de acordo com as recomendações das Diretrizes Metodológicas para Estudos de Avaliação Econômica de Tecnologias em Saúde, publicadas pelo Ministério da Saúde (Ministério da Saúde - Brasil. Secretaria de Ciência, Tecnologia e Insumos Estratégicos. Departamento de Ciência e Tecnologia, 2014).

Tabela 3. Custo unitário dos medicamentos comparadores

\begin{tabular}{lcccc}
\hline Medicamentos & Apresentação & Preço & Posologia (9) & Fonte \\
\hline Obinutuzumabe & $\begin{array}{l}1000 \mathrm{mg} \mathrm{sol} \mathrm{dil} \\
\text { infs IV } 40 \mathrm{~mL}\end{array}$ & $\mathrm{R} \$ 12.506,22$ & $\begin{array}{c}\text { Ciclo 1: 1000 mg } \\
\text { nos dias 1, 8 e 15. } \\
\text { Ciclos 2-6: 1000 mg } \\
\text { no dia 1 }\end{array}$ & CMED - PMVG 0\% \\
\hline APAC (03.04.03.005-8) & $\begin{array}{c}\text { Quimioterapia de } \\
\text { leucemia linfocítica } \\
\text { crônica - 1a linha }\end{array}$ & R\$ 407,50 & Mensal & SIGTAP (03.04.03.005-8) \\
\hline
\end{tabular}


Tabela 4. Custo de acompanhamento mensal

\begin{tabular}{|c|c|c|c|c|c|c|}
\hline & $\%$ em uso & Quantidade & Custo unitário & Custo total & Fonte (16) & Código \\
\hline Consulta médica & $100 \%$ & 2 & $R \$ 10,00$ & $R \$ 20,00$ & SIGTAP 09/2015 & 03.01.01.007-2 \\
\hline Hemograma & $100 \%$ & 0,17 & $\mathrm{R} \$ 4,11$ & $\mathrm{R} \$ 0,69$ & SIGTAP 09/2015 & 02.02.02.038-0 \\
\hline Contagem de plaquetas & $100 \%$ & 0,17 & $R \$ 2,73$ & $\mathrm{R} \$ 0,46$ & SIGTAP 09/2015 & 02.02.02.002-9 \\
\hline Biópsia de medula óssea & $100 \%$ & 0,17 & $R \$ 402,72$ & $\mathrm{R} \$ 67,12$ & TABNET 2013 & $02.01 .01 .027-5$ \\
\hline $\begin{array}{l}\text { Tomografia computadorizada } \\
\text { abdome pelve }\end{array}$ & $100 \%$ & 0,17 & $\mathrm{R} \$ 138,63$ & $R \$ 23,11$ & SIGTAP 09/2015 & 02.06.03.003-7 \\
\hline Radiografia de tórax & $100 \%$ & 0,17 & $R \$ 9,50$ & $R \$ 1,58$ & SIGTAP 09/2015 & $02.04 .03 .015-3$ \\
\hline $\begin{array}{l}\text { Tomografia computadorizada } \\
\text { de abdome }\end{array}$ & $100 \%$ & 0,17 & $\mathrm{R} \$ 138,63$ & $R \$ 23,11$ & SIGTAP 09/2015 & 02.06.03.001-0 \\
\hline TOTAL & & & & $R \$ 136,05$ & & \\
\hline
\end{tabular}

Tabela 5. Custo unitário dos eventos adversos

\begin{tabular}{lc}
\hline Eventos & Custo por evento \\
\hline Anemia & $R \$ 310,00$ \\
\hline Neutropenia febril & $R \$ 202,00$ \\
\hline Infecção & $R \$ 84,00$ \\
\hline Reação relacionada à infusão & $R \$ 10,00$ \\
\hline Leucopenia & $R \$ 269,00$ \\
\hline Neutropenia & $R \$ 202,00$ \\
\hline Pneumonia & $R \$ 84,00$ \\
\hline Trombocitopenia & $R \$ 312,00$ \\
\hline
\end{tabular}

\section{Resultados}

\section{GQT vs. QT}

A comparação de GQT vs. QT resultou em um custo incremental de R\$ 72.565 com 2,2 anos de vida livre de progressão e 1,4 AVG, resultando em uma razão de custo-efetividade incremental (RCEI) de R\$32.477 por ano de vida livre de progressão e R\$ 52.252 por ano de vida ganho (Tabela 6).

\section{Análise de sensibilidade}

Uma análise de sensibilidade univariada e probabilística foi realizada com o intuito de avaliar as incertezas do modelo, por meio da variação de determinados parâmetros. Para ambas as análises, o desfecho de AVG foi o selecionado.

Para a análise de sensibilidade univariada, os parâmetros foram variados $\pm 10 \%$. Os resultados da análise de sensibilidade estão demonstrados na Tabela 7.

Os resultados obtidos demonstraram que os parâmetros que apresentaram maior influência na RCEI de AVG foram a alteração da curva paramétrica para Weibull ou exponencial, e a taxa de desconto dos desfechos clínicos.

No caso da análise de sensibilidade probabilística, os parâmetros foram variados simultaneamente em 1.000 iterações, e os respectivos dados foram avaliados. Os resultados estão demonstrados na Figura 4. Esses valores confirmam os resultados da análise determinística, já que 95\% dos valores se localizaram no quadrante de maior custo e maior benefício clínico, e os 5\% dos resultados restantes permaneceram no quadrante de menor custo e maior benefício clínico.

\section{Discussão}

O objetivo do estudo foi avaliar a custo-efetividade de GQT quando comparado à terapia padrão disponível no SUS para o tratamento da LLC (QT). Os desfechos clínicos avaliados foram o aumento da SG e SLP do paciente dentro do horizonte de tempo de 20 anos (assumido como lifetime). Para ambos os desfechos, GQT apresentou ganhos significativos em anos de vida e anos de vida livres de progressão (1,4 e 2,2 anos, respectivamente) com custo incremental de aproximadamente $\mathrm{R} \$ 72$ mil reais. Esses resultados levam a RCEls de cerca de $R \$ 52$ mil e R\$ 32 mil por AVG e AVLP, respectivamente.

A determinação da custo-efetividade de uma tecnologia em saúde tem, tradicionalmente, sido definida por meio de limiares de custo-efetividade (Marseille et al., 2015), porém, novas alternativas, que apresentam por objetivo oferecer uma visão global e menos determinística do assunto, vêm sendo discutidas. No Brasil, a Comissão Nacional de Avaliações de Tecnologia (CONITEC) não possui um limiar de custo-efetividade determinado, o que demonstra que o assunto permanece como um ponto de discussão em aberto no país e reflete o consenso internacional de que novas alternativas à análise de limiar devem ser propostas. Um estudo publicado em 2015 por Zimmerman et al. (Zimmermann et al., 2015) com o objetivo de determinar os principais fatores preditivos da incorporação de uma tecnologia no SUS classificou, por meio de uma análise de quartis, limiares de custo-efetividade, com base em 67 submissões à CONITEC de pedidos de incorporação de medicamentos, e determinou um limiar máximo de aproximadamente R\$ 70 mil. Apesar de a recomendação de 2002, da OMS, especificar que o limiar de custo-efetividade deve ser empregado para o desfecho de anos de vida ajustados à incapacidade (DALYS) (Marseille et 
Tabela 6. Resultados de custo e efetividade da comparação de GQT vs. QT

\begin{tabular}{|c|c|c|c|}
\hline Desfechos & $\begin{array}{c}\text { Obinutuzumabe }+ \\
\text { Quimioterapia }\end{array}$ & Quimioterapia & Incremental \\
\hline \multicolumn{4}{|l|}{ Custos } \\
\hline Tratamento medicamentoso & $R \$ 86.756$ & $R \$ 1.899$ & \\
\hline Quimioterapia & $\mathrm{R} \$ 1.947$ & - & \\
\hline Administração & $R \$ 306$ & $\mathrm{R} \$ 0,00$ & \\
\hline Acompanhamento & $R \$ 5.459$ & $\mathrm{R} \$ 1.812$ & \\
\hline Eventos adversos & $\mathrm{R} \$ 154$ & $\mathrm{R} \$ 80$ & \\
\hline Média de custo na SLP & $\mathrm{R} \$ 94.622$ & $\mathrm{R} \$ 3.791$ & $\mathrm{R} \$ 90.831$ \\
\hline Média de custo na progressão & $\mathrm{R} \$ 50.871$ & $\mathrm{R} \$ 69.136$ & - R\$ 18.265 \\
\hline Total & $\mathrm{R} \$ 145.492$ & $\mathrm{R} \$ 72.927$ & $\mathrm{R} \$ 72.565$ \\
\hline \multicolumn{4}{|l|}{ Efetividade } \\
\hline Sobrevida livre de progressão & 3,344 & 1,110 & 2.234 \\
\hline RCEI (R\$/SLP) & & & $\mathrm{R} \$ 32.477$ \\
\hline Anos de vida ganhos & 5,699 & 4,310 & 1,389 \\
\hline RCEI (R\$/AVG) & & & $R \$ 52.252$ \\
\hline
\end{tabular}

RCEl: razão de custo-efetividade incremental; SLP: sobrevida livre de progressão.

Tabela 7. Parâmetros variados na análise de sensibilidade

\begin{tabular}{|c|c|c|c|c|}
\hline & Caso base & Limite inferior & Limite superior & Diferença* \\
\hline Curva paramétrica - Weibull & $R \$ 52.252$ & $\mathrm{R} \$ 62.722$ & & \\
\hline Curva paramétrica - Exponencial & $R \$ 52.252$ & $R \$ 53.171$ & & \\
\hline Taxa de desconto (desfechos) & $\mathrm{R} \$ 52.252$ & $\mathrm{R} \$ 50.703$ & $\mathrm{R} \$ 53.819$ & $R \$ 3.116$ \\
\hline Idade média & $R \$ 52.252$ & $R \$ 55.706$ & $R \$ 52.445$ & $R \$ 3.261$ \\
\hline Taxa de desconto (custos) & $R \$ 52.252$ & $\mathrm{R} \$ 52.311$ & $\mathrm{R} \$ 52.200$ & $\mathrm{R} \$ 111$ \\
\hline Eventos adversos (obinutuzumabe) & $\mathrm{R} \$ 52.252$ & $\mathrm{R} \$ 52.241$ & $R \$ 52.263$ & $R \$ 22$ \\
\hline Eventos adversos (quimioterapia) & $R \$ 52.252$ & $\mathrm{R} \$ 52.258$ & $\mathrm{R} \$ 52.246$ & $\mathrm{R} \$ 12$ \\
\hline
\end{tabular}

* Diferença entre limite superior e limite inferior.

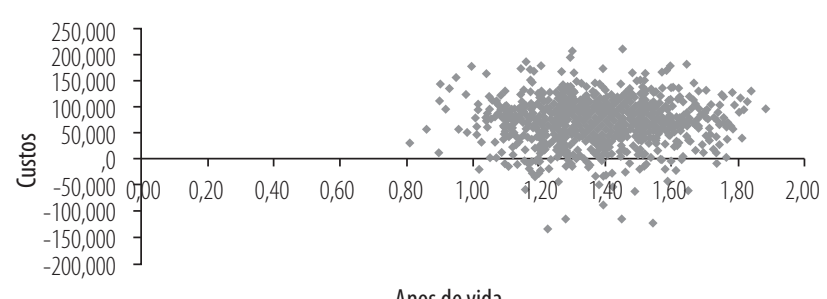

Anos de vida

Figura 4. Resultados da análise de sensibilidade probabilística.

al., 2015), a análise da publicação em questão não fez restrição ao tipo de desfecho utilizado para sua classificação. Com base nessa avaliação, pode-se afirmar que GQT mostrou-se custo-efetivo, uma vez que GQT apresentou resultados de custo-efetividade similares às tecnologias incorporadas ao SUS, analisadas previamente pela CONITEC.
Ainda em relação à publicação de Zimmerman et al., o estudo demonstrou que o preditivo mais forte para a incorporação de um medicamento ao SUS é o grau de qualidade da evidência apresentada à CONITEC (Zimmermann et al., 2015). Nesse sentido, a publicação do estudo CLL11 apresenta o maior grau de recomendação possível dentro da escala do Oxford-Center for Evidence Based Medicine (1B/A). Esse fator é importante, pois, além de garantir a confiabilidade dos achados dos estudos clínicos, ele garante que as extrapolações dos dados para um horizonte de tempo superior, por meio de modelagem econômica, sejam realistas e baseadas em evidências clínicas fortes.

Adicionalmente, no âmbito internacional, alguns estudos também apresentaram resultados favorecendo a associação de GClb no tratamento de pacientes LLC. De acordo com Casado et al., sob perspectiva do sistema de saúde espanhol, a combinação de GClb em comparação ao rituximabe associado a clorambucila apresentou benefícios clínicos em anos de vida e QALY, além de poder ser classificada como efetiva em 
comparação aos limiares comumente utilizados na Espanha (Casado et al., 2016). Adicionalmente, em um estudo europeu, cujo objetivo foi avaliar a custo-efetividade de tratamentos de primeira linha de LLC para pacientes inelegíveis a fludarabina, concluiu-se que GClb demonstrou ser a melhor opção com relação clínica e valor para custo-efetividade (Soini et al., 2016), além de outros estudos realizados no Reino Unido e Holanda que também corroboraram com os achados das outras análises de custo-efetividade mencionadas anteriormente (Becker et al., 2016; Blommestein et al., 2016). Por meio dessas informações, pode-se concluir que os resultados desse estudo corroboram resultados obtidos em outros países, mesmo quando adequados a suas próprias realidades.

Em adição a essas evidências, algumas agências de avaliação de tecnologias em saúde internacionais recomendam a utilização de GClb em pacientes com LLC não tratados previamente, com comorbidades e inelegíveis ao tratamento com fludarabina, devido a seus resultados clínicos e econômicos favoráveis. Dentre essas instituições estão o Canadian Agency for Drugs and Technologies in Health Pan-Canadian Oncology Drug Review (CADTH - pCODR) e The National Institute for Health and Care Excellence (NICE) (National Institute for Health and Clinical Excellence - NICE, 2015; Pan-Canadian Oncology Drug Review - pCODR. Canadian Agency for Drugs and Technologies in Health - CADTH, 2015). Assim, ratifica-se que a associação GClb representa uma opção terapêutica de impacto em um contexto de necessidades médicas não atendidas para o tratamento de pacientes com LLC, com comorbidade e inelegíveis ao tratamento com fludarabina.

Entretanto, vale ressaltar que os dados utilizados no modelo podem não refletir a realidade clínica, uma vez que as informações e valores para os cálculos foram retirados de estudos clínicos, cujos protocolos, incluindo exames e regimes de tratamento, foram monitorados e controlados. Por isso, essas informações não podem ser interpretadas, necessariamente, como reflexo do mundo real. Em adição a isso, a metodologia simples de modelagem com três estados de saúde pode representar uma forma limitante de refletir uma doença crônica, uma vez que, em um período de lifetime, o paciente pode apresentar outros estados de saúde, além de sofrer influências de linhas posteriores de tratamento. A ausência de dados que apresentem o acompanhamento de pacientes em todas as fases da doença também é um fator que dificulta as estimativas de desfechos clínicos para avaliações econômicas (Marsh, et al., 2014).

Além disso, outra limitação do modelo que deve ser descrita é a utilização da curva do estudo CLL5 para o cálculo das probabilidades de pacientes que transitaram do estado de pós-progressão para a morte. Na ausência desses dados no estudo CLL11, foi necessário realizar um ajuste para adequar as informações do estudo CLL5 aos cálculos dessa análise.
Apesar dos aspectos limitantes, esse estudo apresenta grande importância para a estimativa da razão de custo-efetividade incremental de GQT em comparação à QT para avaliações econômicas no tratamento de LLC no Brasil. Mesmo na ausência de informações a longo prazo, a maioria dos estudos com modelos econômicos para LLC adota o mesmo formato de modelagem do presente estudo, e foi e é aceita por agências de tecnologia de saúde internacionais (Marsh et al., 2014). A utilização de análise de custo-efetividade auxilia na comparação tanto de desfechos clínicos quanto de desfechos econômicos de duas intervenções, além de serem ferramentas importantes e úteis durante a tomada de decisão de fontes pagadoras, principalmente em um contexto de recursos econômicos limitados.

\section{Conclusão}

Atualmente, imunoquimioterapia com FCR é o padrão de tratamento em pacientes com LLC sem tratamento anterior que precisam de tratamento e têm boas condições físicas para receber QT intensa (Diretrizes NCCN, 2013; SBOC, 2011) (Eichhorst et al., 2011; Zelenetz et al., 2013). Porém, a maioria dos pacientes com LLC tem idade avançada e frequentemente apresenta condições médicas concomitantes e/ou diminuição da função de algum órgão que os impede de receber o padrão de tratamento comum (FCR). Dessa forma, são necessárias alternativas terapêuticas.

Nesse sentido e considerando ainda que os sistemas de saúde enfrentam desafios em suas gestões decorrentes do aumento crescente da utilização de recursos e gastos, a adoção de opções terapêuticas economicamente favoráveis é a mais recomendada no contexto de saúde atual. Dessa forma, são necessárias alternativas terapêuticas que visem à integralidade da assistência farmacêutica de pacientes com LLC e, sobretudo, a sustentabilidade do sistema.

A presente análise concluiu que a terapia GQT é uma opção terapêutica que proporciona benefícios superiores quando comparada à QT e pode ser considerada custo-efetiva no tratamento de LLC em pacientes não elegíveis a doses completas de fludarabina na perspectiva do SUS.

\section{Referências bibliográficas}

Becker U, Briggs AH, Moreno SG, Ray JA, Ngo P, Samanta K. Cost-Effectiveness Model for Chemoimmunotherapy Options in Patients with Previously Untreated Chronic Lymphocytic Leukemia Unsuitable for Full-Dose Fludarabine-Based Therapy. Value Heal. 2016;19(4):374-82.

Blommestein HM, de Groot S, Aarts MJ, Vemer P, de Vries R, van Abeelen AFM, et al. Cost-effectiveness of obinutuzumab for chronic lymphocytic leukaemia in The Netherlands. Leuk Res. 2016:50:37-45.

Casado LF, Burgos A, González-Haba E, Loscertales J, Krivasi T, Orofino J, et al. Economic evaluation of obinutuzumab in combination with chlorambucil in first-line treatment of patients with chronic lymphocytic leukemia in Spain. Clinicoecon Outcomes Res. 2016;8:475-84. 
Cramer P, Hallek M. Prognostic factors in chronic lymphocytic leukemia-what do we need to know? Nat Rev Clin Oncol. 2011;8(1):38-47.

Eichhorst B, Dreyling M, Robak T, Montserrat E, Hallek M. Chronic lymphocytic leukemia: ESMO clinical practice guidelines for diagnosis, treatment and follow-up. Ann Oncol. 2011; 22(Suppl. 6):vi50-4.

Eichhorst BF, Busch R, Stilgenbauer S, Stauch M, Bergmann MA, Ritgen M, et al. First-line therapy with fludarabine compared with chlorambucil does not result in a major benefit for elderly patients with advanced chronic lymphocytic leukemia. Blood. 2009;114(16):3382-91.

Goede V, Fischer K, Engelke A, Schlag R, Lepretre S, Montero LFC, et al. Obinutuzumab as frontline treatment of chronic lymphocytic leukemia: updated results of the CLL11 study. Leukemia. 2015;1-3.

Hallek M. Chronic lymphocytic leukemia: 2015 Update on diagnosis, risk stratification, and treatment. Am J Hematol. 2015;90(5):44660.

Herishanu Y, Polliack A. Chronic lymphocytic leukemia: a review of some new aspects of the biology, factors influencing prognosis and therapeutic options. Transfus Apher Sci. 2005;32(1):85-97.

Hillmen P, Gribben JG, Follows GA, Milligan D, Sayala HA, Moreton P, et al. Rituximab plus chlorambucil as first-line treatment for chronic lymphocytic leukemia: Final analysis of an open-label phase II study. J Clin Oncol. 2014;32(12):1236-41.

Instituto Brasileiro de Geografia e Estatística (IBGE). Antropometria e estado nutricional de crianças, adolescentes e adultos no Brasil [Internet]. Disponível em: http://www.ibge.gov.br/home/estatistica/populacao/ condicaodevida/pof/2008_2009_encaa/defaulttabpdf_brasil.shtm. Acesso em: 2 fev. 2015.

Instituto Brasileiro de Geografia e Estatística (IBGE). Tábua de mortalidade. [Internet]. 2012. Disponível em: http://www.ibge.gov.br/home/ estatistica/populacao/tabuadevida/2012/. Acesso em: 26 fev. 2015.

Marseille E, Larson B, Kazi DS, Kahn JG, Rosen S. Thresholds for the costeffectiveness of interventions: alternative approaches. Bull World Health Organ. 2015;93(2):118-24.

Marsh K, Xu P, Orfanos P, Gordon J, Griebsch I. Model-Based CostEffectiveness Analyses for the Treatment of Chronic Lymphocytic Leukaemia: A Review of Methods to Model Disease Outcomes and Estimate Utility. Pharmacoeconomics. 2014;32(10):981-93.

Ministério da Saúde (Brasil). Agência Nacional de Vigilância Sanitária (ANVISA). Câmara de Regulação do Mercado de Medicamentos (CMED). [Internet]. 2016. Disponível em: http://portal.anvisa.gov.br/cmed. Acesso em: 26 fev. 2015.

Ministério da Saúde (Brasil). Departamento de Informática do SUS (DATASUS). [Internet]. 2016. Disponível em: http://www2.datasus.gov.br/DATASUS/ index.php?area=. Acesso em: 26 fev. 2015.
Ministério da Saúde (Brasil). Sistema de Gerenciamento da Tabela de Procedimentos (SIGTAP). Competência Outubro de 2011. [Internet]. 2011. Disponível em: http://sigtap.datasus.gov.br/tabela-unificada/app/sec/ inicio.jsp2011. Acesso em: 26 fev. 2015.

Ministério da Saúde (Brasil). Secretaria de Ciência-Tecnologia e Insumos Estratégicos. Departamento de Ciência e Teccnologia. Diretrizes metodológicas: estudos de avaliação econômica de tecnologias em saúde. 2nd ed. Brasília: Ministério da Saúde; 2014. 132 p.

Ministério da Saúde (Brasil). Instituto Nacional de Câncer José Alencar Gomes da Silva (INCA). Leucemia - Subtipos [Internet]. 2015. Disponível em: http://www2.inca.gov.br/wps/wcm/connect/tiposdecancer/site/ home/leucemia/subtipos. Acesso em: 26 fev. 2015.

National Comprehensive Cancer Network (NCCN). Non-Hodgkin's Lymphomas - Version 2013.

National Institute for Health and Clinical Excellence (NICE). Obinutuzumab in combination with chlorambucil for untreated chronic lymphocytic leukaemia [Internet]. 2015. Disponível em: https://www.nice.org.uk/ guidance/ta343/chapter/4-Consideration-of-the-evidence\#summary-ofappraisal-committees-key-conclusions. Acesso em: 26 fev. 2015.

Pan-Canadian Oncology Drug Review (pCODR). Canadian Agency for Drugs and Technologies in Health (CADTH). pCODR Final Clinical Guidance Report - Obinutuzumab (Gazyva) for Chronic Lymphocytic Leukemia. 2015;63.

Produtos Roche Químicos e Farmacêuticos S.A. Gazyva (obinutuzumab). 2015. p. 1-23.

Rozman C, Montserrat E. Chronic lymphocytic leukemia. N Engl J Med. 1995;333(16):1052-7.

Shvidel L, Shtalrid M, Bairey O, Rahimi-Levene N, Lugassy G, Shpilberg O, et al. Conventional dose fludarabine-based regimens are effective but have excessive toxicity in elderly patients with refractory chronic lymphocytic leukemia. Leuk Lymphoma. 2003:44(11):1947-50.

Sociedade Brasileira de Oncologia Clínica (SBOC). Manual de Condutas 2011. Rev Soc Bras Oncol Clínica. 2011;589.

Soini E, Hautala A, Poikonen E, Becker U, Kyttälä M, Martikainen J. Costeffectiveness of First-line Chronic Lymphocytic Leukemia Treatments When Full-dose Fludarabine Is Unsuitable. Clin Ther. 2016;38(4):889-904.e14.

Zelenetz AD, Wierda WG, Abramson JS, Advani RH, Andreadis CB, Bartlett $\mathrm{N}$, et al. Non-Hodgkin's lymphomas, version 1.2013. J Natl Compr Canc Netw. 2013;11(3):257-72; quiz 273.

Zimmermann IR, Oliveira EF de, Vidal AT, Santos VCC, Petramale CA. A qualidade das evidências e as recomendações sobre a incorporação de medicamentos no sistema único de saúde: uma análise retrospectiva. Rev Eletronica Gestão Saúde. 2015;6(4):3043. 